\title{
Uno studio diagnostico multicentrico sul nodulo tiroideo basato su un algoritmo generato da un modello di intelligenza artificiale
}

\author{
Carmelo Gusmano $^{1}$ - Aldo E. Calogero ${ }^{1}$
}

Accettato: 30 giugno 2021 / Pubblicato online: 8 settembre 2021

(c) The Author(s) 2021

Commento a:

Deep learning-based artificial intelligence model to assist thyroid nodule diagnosis and management: a multicentre diagnostic study.

S. Peng, Y. Liu, W. Lv, L. Liu, Q. Zhou, H. Yang, J. Ren, G. Liu, X. Wang, X. Zhang, Q. Du, F. Nie, G. Huang, Y. Guo, J. Li, J. Liang, H. Hu, H. Xiao, Z. Liu, F. Lai, Q. Zheng, H. Wang, Y. Li, E.K. Alexander, W. Wang, H. Xiao.

Lancet Digit Health (2021) 3(4):e250-e259

La tireopatia nodulare interessa dal 50 al $60 \%$ della popolazione sana [1]. La maggior parte dei pazienti affetti dalla patologia nodulare non lamenta sintomi clinici significativi e, pertanto, la gestione clinica dipende dalle caratteristiche ecografiche e dall'esame citologico eseguito su aspirazione del nodulo con ago sottile (FNA). Nel 7-15\% dei casi, il nodulo è un carcinoma tiroideo che rappresenta la patologia maligna più rapidamente in crescita nella popolazione generale [2]. Il vasto numero di noduli tiroidei, dei quali solo una piccola parte è di natura tumorale, richiede metodi affidabili per differenziare accuratamente i noduli maligni da quelli benigni [2]. Le linee guida stratificano i noduli tiroidei in base alle caratteristiche ecografiche in [1]: 1) noduli a basso rischio, quando cistici ( $>50 \%$ ) con artefatti di riverbero non sospetti o isoecogeni spongiformi confluenti o con alone regolare; 2) noduli a rischio intermedio, quando leggermente ipoecogeni o isoecogeni con forma ovalare/circolare e margini regolari o mal definiti, vascolarizzazione intranodulare, elevata rigidità all'elastosonografia, macrocalcifica-

$凶$ A.E. Calogero

acaloger@unict.it

1 Dipartimento di Medicina Clinica e Sperimentale, Università di Catania, Catania, Italia zioni o rima calcifica continua; e 3) noduli a rischio elevato, con ipoecogenicità marcata (uguale ai muscoli pretiroidei), margini spigolati o microlobulati, microcalcificazioni e taller-than-wide.

Numerosi sforzi sono stati compiuti ad oggi nel tentativo di migliorare l'accuratezza diagnostica della tireopatia nodulare, in modo da discriminare al meglio tra patologia nodulare benigna e carcinoma tiroideo. Ne sono esempi gli studi di biologia molecolare aventi lo scopo di indentificare marcatori molecolari predittivi di patologia oncologica. $\mathrm{Nel}$ recente periodo però, sembra che anche l'intelligenza artificiale (IA) possa avere un ruolo.

È stato riportato che l'IA sia in grado di eguagliare o superare gli esperti in diagnostica morfologica e per immagini. Tuttavia, solo pochi studi hanno valutato le prestazioni diagnostiche dell'IA con quelle umane nella differenziazione dei noduli tiroidei [2]. Un recente studio diagnostico multicentrico ha sviluppato un algoritmo informatico con deeplearning (ThyNet) per differenziare i tumori maligni dai noduli benigni e valutare se questo strumento aumenti l'accuratezza diagnostica cercando anche di ridurre il numero di FNA non necessarie [2]. Per il periodo di training, sono state selezionate 18.049 immagini di 8339 pazienti. I casi selezionati hanno poi ricevuto una diagnosi istologica o citologica definitiva. Successivamente, gli autori hanno eseguito tre fasi di studio differenti. Nel Test A (2185 immagini di 1424 pazienti), l'accuratezza diagnostica del ThyNet è stata confrontata con quella dei radiologi. Nel Test B (1754 immagini di 1048 pazienti), gli autori hanno valutato il miglioramento della performance diagnostica dei radiologi quando assistiti dal ThyNet, permettendo loro di rivalutare il giudizio diagnostico dopo aver consultato la diagnosi espressa dall'algoritmo ThyNet. Fino a questo punto dello studio, le immagini e i pazienti sono stati arruolati retrospettivamente. Nel Test C (366 immagini di 303 pazienti), è stata indagata l'ap- 
plicazione del ThyNet nella pratica clinica reale, in maniera prospettica, registrando le differenze tra l'accuratezza diagnostica dei radiologi dopo aver visto le immagini, successivamente ad aver visionato dei video dinamici dei noduli e, infine, dopo aver consultato la diagnosi del ThyNet. Gli autori hanno scelto come end-point primario l'area under the receiver operating characteristic curve della diagnosi del nodulo tiroideo. Gli end-point secondari erano l'accuratezza, la specificità, la sensibilità, il valore predittivo positivo e negativo di ThyNet. In tutte le fasi dello studio (A, B e C), si è registrato un miglioramento statisticamente significativo dei parametri analizzati, maggiormente evidente quando l'algoritmo ThyNet è stato impiegato con radiologi junior (con esperienza da uno a tre anni) rispetto ai radiologi senior (con più di otto anni esperienza) [2].

Questo è il primo studio che valuta una strategia basata sul deep-learning come ausilio per la gestione del nodulo tiroideo. I risultati dimostrano che l'applicazione del ThyNet migliori l'accuratezza diagnostica dei radiologi sia in maniera retrospettiva che prospettica. Inoltre, integrando la classificazione ACR TI-RADS che, ad oggi, riporta il più basso tasso di FNA non necessarie, con l'assistenza dell'IA si è ottenuto un aumento del valore predittivo negativo e positivo che potrebbe permettere un'ulteriore riduzione del numero di FNA non necessarie [2].

Funding Note Open access funding provided by Università degli Studi di Catania within the CRUI-CARE Agreement.
Nota della casa editrice Springer Nature rimane neutrale in riguardo alle rivendicazioni giurisdizionali nelle mappe pubblicate e nelle affiliazioni istituzionali.

Open Access This article is licensed under a Creative Commons Attribution 4.0 International License, which permits use, sharing, adaptation, distribution and reproduction in any medium or format, as long as you give appropriate credit to the original author(s) and the source, provide a link to the Creative Commons licence, and indicate if changes were made. The images or other third party material in this article are included in the article's Creative Commons licence, unless indicated otherwise in a credit line to the material. If material is not included in the article's Creative Commons licence and your intended use is not permitted by statutory regulation or exceeds the permitted use, you will need to obtain permission directly from the copyright holder. To view a copy of this licence, visit http://creativecommons.org/licenses/by/4.0/.

\section{Bibliografia}

1. Gharib H, Papini E, Garber JR et al (2016) Medical guidelines for clinical practice for the diagnosis and management of thyroid nodules-2016 Update. Endocr Pract 22(5):622-639

2. Peng S, Liu Y, Lv W et al (2021) Deep learning-based artificial intelligence model to assist thyroid nodule diagnosis and management: a multicentre diagnostic study. Lancet Digit Health 3(4):e250-e259 